\title{
Social Determinants and COVID-19 in a Community Health Center Cohort
}

\author{
Moid Ali $^{1,2} \cdot$ Victoria Gasca $^{1} \cdot$ Rachel Schrier $^{1,3} \cdot$ Mellisa Pensa $^{1,3} \cdot$ Anthony Brockman $^{1} \cdot$ Douglas P. Olson $^{1}$. \\ Benjamin J. Oldfield ${ }^{1,3}$
}

Accepted: 23 November 2021 / Published online: 30 November 2021

(c) The Author(s), under exclusive licence to Springer Science+Business Media, LLC, part of Springer Nature 2021

\begin{abstract}
Associations between social determinants of health (SDOH), demographic factors including preferred language, and SARS$\mathrm{CoV}-2$ detection are not clear. We conducted a retrospective cohort study among those seeking testing for SARS-CoV-2 at a multi-site, urban community health center. Logistic regression and exact matching methods were used to identify independent predictors of SARS-CoV-2 detection among demographic, SDOH, and neighborhood-level variables. Of 1,361 included individuals, SARS-CoV-2 was detected among 266 (19.5\%). Logistic regression demonstrated that SARS-CoV-2 detection was less likely in White participants relative to Hispanic participants (adjusted odds ratio [aOR] 0.18, 95\% confidence interval [CI] 0.05-0.46). and more likely in patients who prefer Spanish relative to those that prefer English (aOR 2.04, 95\% CI 1.43-2.96). No observed SDOH predicted SARS-CoV-2 detection in adjusted models. A robustness analysis using a matched subset of the study sample produced findings similar to those in the main analysis. Preferring to receive care in Spanish is an independent predictor of SARS-CoV-2 detection in a community health center cohort.
\end{abstract}

Keywords SARS-CoV-2 $\cdot$ COVID-19 $\cdot$ Social determinants of health $\cdot$ Community health centers

\section{Background}

The coronavirus disease 2019 (COVID-19) pandemic has revealed opportunities for public health and primary care infrastructure to better target the social and structural determinants of health, and to promote health equity [1]. Disparities in incidence and mortality of COVID-19 have been documented by race/ethnicity [2] and socioeconomic status $[3,4]$. These hierarchies pose intersecting vulnerabilities on individuals and communities that drive poorer health outcomes through the social determinants of health (SDOH), the conditions in which individuals live that impact health and wellbeing $[5,6]$.

Benjamin J. Oldfield

benjamin.oldfield@yale.edu

1 Fair Haven Community Health Care, 374 Grand Avenue, New Haven, CT 06513, USA

2 Department of Epidemiology of Microbial Diseases, Yale School of Public Health, New Haven, CT 06510, USA

3 Department of Medicine, Yale School of Medicine, New Haven, CT 06510, USA
While the impacts of SDOH on COVID-19 incidence and outcomes have been described, how individual factors may independently drive the risk of infection has not. Previous studies have focused on specific ethnic populations [7] or individual sociostructural phenomena such as school closures in impoverished areas [8], neighborhood deprivation [9], homelessness [10], and food insecurity [3], demonstrating higher risk for COVID-19 among groups experiencing particular vulnerabilities. However, the comparative effect of demographic and SDOH variables at individual and neighborhood levels requires further elucidation so that public health programming, health care organizations, and community health workers can prioritize efforts towards patients and communities at highest risk. Calls have been made for quantifying and characterizing the harmful social conditions that fundamentally shape pandemic patterns to inform public health interventions targeting COVID-19 [11, 12].

Community health centers (CHCs), which serve as primary medical homes for more than 28 million Americans, are an ideal setting for the assessment of social needs and linkage to services for families [13]. In addition to providing care that includes services often otherwise unavailable to low-income and uninsured populations [14], 
they also represent a social movement whose focus has historically been on meeting social needs through community partnerships [15]. Major societies representing clinicians who provide pediatric, perinatal, and family care in CHCs, including the American Academy of Family Physicians, the American Academy of Pediatrics, and the American College of Obstetricians and Gynecologists, have recommended screening for SDOH during medical visits using standardized assessment tools [16-18], yet individual clinicians and $\mathrm{CHCs}$ may be unsure how to act on this data [19] and how it may impact COVID-19 risk. Our aim was to identify independent predictors-among sociodemographic, $\mathrm{SDOH}$, and neighborhood-level variables-of SARS-CoV-2 infection among a CHC cohort seeking walk-up testing free of charge and regardless of insurance status.

\section{Theoretical Framework}

The Healthy People 2030 Social Determinants of Health Framework [20], which includes domains on economic stability, education, social and community context, health and health care, and neighborhood and built environment, organizes potential targets of public health intervention to improve upon the unequal distribution of SDOH [11].

\section{Methods}

\section{Participants}

We conducted a retrospective cohort study of patients seeking walk-up SARS-CoV-2 polymerase chain reaction (PCR) testing at Fair Haven Community Health Care, a federally qualified community health center in New Haven, CT. Ninety percent of the patients cared for by the health center have an income that is less than $200 \%$ of the Federal Poverty Level and self-identify as a racial or ethnic minority. Nearly fifty percent of patients cared for by the health center prefer to receive care in a language other than English. Free walk-up testing was made available to all regardless of insurance status, legal residency, and current display of symptoms. SARS-CoV-2 test results ranging between March and December 2020 were included in our study sample. We included participants who completed both a SARS-CoV-2 test and a SDOH screen either at the point of testing or at any prior episode of medical care at Fair Haven Community Health Center after 2018. We excluded patients whose preferred language was neither English nor Spanish.

\section{Measures}

SDOH data were collected using the Accountable Health Community (AHC) Health Related Social Needs Tool [21]. The AHC tool assesses unmet social need across five domains: housing, food, transportation, utilities, and interpersonal safety. For our analysis, we excluded questions regarding interpersonal safety due to incompleteness. We collapsed the remaining questions to four binary indicators for housing inadequacy, food insecurity, unreliable transportation, and utility needs. The collapsed housing inadequacy indicator captures respondents who are worried about losing their current residence, do not have a current stable residence, or report any housing quality problems such as water leaks, pests, or mold at the time of the survey. The food insecurity indicator captures respondents who are worried about running out of food in the past 12 months. Similarly, the unreliable transportation and utility needs captured unmet need in these domains in the past 12 months. Positive responses were those that indicated any amount of unmet social need within these four categories. The AHC tool was conducted using the individual respondent's self-indicated preferred language with the assistance of a telephonic interpreter as needed.

We categorized patients as living in socially deprived neighborhood using the Social Deprivation Index (SDI), developed by the Robert Graham Center [22]. The SDI provides a within-state index ranging from 1 , the least disadvantaged, to 100 , the most disadvantaged for Zip Code geographies. The SDI incorporates demographic and socioeconomic measures across seven domains sourced from the American Community Survey 2011-2015 5-year estimates. Participants were considered to live in a socially deprived neighborhood if their 5-digit residential Zip Code fell within the top SDI quartile in Connecticut.

Our outcome measure was the detection of SARSCoV-2 by PCR via nasopharyngeal or anterior nasal swab. Participants with multiple SARS-CoV-2 PCR tests were considered positive if any of the tests returned a positive result within the study period. Self-reported sociodemographic factors included age, sex, race and ethnicity, insurance coverage, neighborhood of residence, preferred language, and $\mathrm{SDOH}$ as measured by the AHC tool. All data were abstracted from the individual participant's electronic medical record.

\section{Analysis}

We examined the distribution of sociodemographic characteristics between SARS-CoV-2 detection groups. Statistical difference in age distribution was evaluated using 
a two-sided independent t-test, assuming unequal variances. Chi-squared tests were used to assess significance of SARS-CoV-2 detection frequency by sex, race and ethnicity, preferred language, insurance, SDOH exposure and neighborhood of residence. Logistic regression was used to determine unadjusted and adjusted odds ratios associated with demographic factors and SARS-CoV-2 detection. $\mathrm{SDOH}$ as a risk factor was assessed using three schemes based on AHC tool responses: high SDOH burden, any positive $\mathrm{SDOH}$, and individual $\mathrm{SDOH}$. Respondents were considered to have high SDOH burden if they had responded positively to two or more of the four included SDOH categories.

Matching analysis was performed to alternatively estimate the odds ratio associated with Spanish language preference, a factor that may be associated with unmeasured social exposures such as employment type or experiences shaped by stigma. Given our interest in language preference, we used matching to isolate variation in language within our study sample and estimate its association with SARS-CoV-2 detection. We used exact matching to identify a subsample of participants with balanced age, sex, race and ethnicity, insurance status, and neighborhood vulnerability but varied language preference. After matching, weighted logistic regression was used to estimate SARS-CoV-2 detection odds between English and Spanish preferred language groups as a robustness check against our main analysis findings.

All analyses were preformed using $\mathrm{R}$ version 3.3.3. Exact matching was performed using the MatchIt package. A two sided $\mathrm{P}<0.05$ determined statistical significance. All study protocols and procedures were reviewed by the Yale School of Medicine Human Investigation Committee.

\section{Results}

During the study period, 1360 individuals were tested for SARS-CoV-2 and completed the AHC tool. Among these, 9 individuals $(0.6 \%)$ preferred to receive care in a language other than English or Spanish and were excluded, for a total study sample of 1351 individuals; this represents $6.8 \%$ of all those who received SARS-CoV-2 testing at the FHCHC during the study period. Among those who underwent testing and completed the AHC tool, in 263 (19.5\%), SARS-COV-2 was detected. Of the total study sample, 1003 (74.2\%) participants were Hispanic, 143 (10.6\%) were Non-Hispanic Black, 160 (11.8\%) were Non-Hispanic White and the remaining $45(3.3 \%)$ were other race or ethnicity, which includes American Indian or Alaskan Native, Native Hawaiian or Other Pacific Islander, Asian, and Multiple Race. 661 (48.9\%) participants had a Spanish language preference while the remaining preferred English. For insurance coverage, $423(31.3 \%)$ participants were uninsured, $716(53.0 \%)$ were publicly insured (including Medicaid and Medicare plans), and 213 (15.7\%) were covered by private or other insurance plans (including worker's compensation and those marked as "other" in the electronic medical record).

Responses to the AHC tool showed 171 (12.7\%) respondents experience inadequate housing, 381 (28.2\%) experience food insecurity, $110(8.1 \%)$ experience unreliable transportation, and 133 (9.8\%) experience difficulty paying for utilities. Aggregating these four categories of SDOH burden, we found that $532(39.4 \%)$ of respondents reported any unmet social need while 204 (15.1\%) reported two or more unmet social needs, meeting our definition for high SDOH burden. $1174(86.9 \%)$ participants were deemed to reside in the top quartile of socially deprived 5-digit Zip Codes, meeting our definition of high residential SDI. Age, race and ethnicity, preferred language, insurance type, food insecurity, and high residential SDI were significantly different between SARSCoV-2 detected and not-detected groups at the $\mathrm{P}<0.05$ level. Sex, indicators for any SDOH and high SDOH burden, and individual indicators of housing instability, utility needs and unreliable transportation were not found to be significantly different between SARS-CoV-2 detected and not-detected groups at the $\mathrm{P}<0.05$ level (Table 1; Fig. 1).

Unadjusted logistic regression analyses show Non-Hispanic White participants having significantly less risk of SARS-CoV-2 detection (odds ratio [OR] 0.09, 95\% confidence interval [CI] 0.03-0.21) relative to Hispanic participants. Non-Hispanic Black (OR 0.64, 95\% CI 0.39-1.01) and other race and ethnicity (OR $0.52,95 \%$ CI $0.19-1.15$ ) participants have indistinguishable risk of SARS-CoV-2 detection relative to Hispanic participants. Those with a Spanish preferred language had higher odds (OR 2.85, 95\% CI 2.15-3.82) of detection relative to those with English as their preferred language. Uninsured participants (OR 2.15, 95\% CI 1.42-3.33) have higher odds of SARS-CoV-2 detection relative to those covered under private or other plans. Food insecurity (OR 1.36, 95\% CI 1.02-1.82) was the only individual $\mathrm{SDOH}$ factor with a significant association of SARS-CoV-2 detection. All other individual factors along with both aggregate measures for SDOH exposure, any SDOH burden (OR 1.20, 0.91-1.58) and high SDOH burden (OR 1.01, 95\% CI 0.69-1.46), had insignificant unadjusted odds of SARS-CoV-2 detection.

The adjusted logistic regression model included sex and all variables with significantly different distributions between SARS-CoV-2 undetected and detected groupsage, race and ethnicity, language preference, insurance coverage type, food insecurity and high residential SDI. In the adjusted model, White participants (adjusted odds ratio [aOR] $0.18,95 \%$ CI 0.05-0.46) remained at significantly reduced risk for SARS-CoV-2 detection relative to Hispanic participants. Patients with a Spanish preferred language had higher odds (aOR 2.04, 95\% CI 1.43-2.96) of detection 
Table 1 Characteristics of Patients Screened for COVID-19 in a community health center cohort $(\mathrm{N}=1351)$

\begin{tabular}{|c|c|c|c|c|c|}
\hline Characteristic & Total $(\mathrm{N}=1351)$ & $\begin{array}{l}\text { COVID-19 not } \\
\text { detected }(\mathrm{N}=1088)\end{array}$ & $\begin{array}{l}\text { COVID-19 } \\
\text { detected }(\mathrm{N}=263)\end{array}$ & $P_{\text {value }}{ }^{d}$ & $\begin{array}{l}\text { Unadjusted odds } \\
\text { ( } 95 \% \text { confidence } \\
\text { interval) }\end{array}$ \\
\hline Age, mean (SD) & $40.26(18.56)$ & $40.81(18.79)$ & $37.98(17.43)$ & 0.02 & $0.99(0.98-1.00)$ \\
\hline Sex, No. (\%) & & & & 0.20 & \\
\hline Male & 562 & $443(78.83 \%)$ & $119(21.17 \%)$ & & $1.20(0.92-1.58)$ \\
\hline Female & 789 & $645(81.75 \%)$ & $144(18.25 \%)$ & & Ref \\
\hline Race and ethnicity, No. (\%) & & & & $<0.001$ & \\
\hline Hispanic & 1003 & $773(77.07 \%)$ & $230(22.93 \%)$ & & Ref \\
\hline Non-Hispanic Black & 143 & $120(83.92 \%)$ & $23(16.08 \%)$ & & $0.64(0.39-1.01)$ \\
\hline Non-Hispanic White & 160 & $156(97.50 \%)$ & $4(2.50 \%)$ & & $0.09(0.03-0.21)$ \\
\hline Other $^{\mathrm{a}}$ & 45 & $39(86.67 \%)$ & $6(13.33 \%)$ & & $0.52(0.19-1.15)$ \\
\hline Language, No. (\%) & & & & $<0.001$ & \\
\hline English & 690 & $609(88.26 \%)$ & $81(11.74 \%)$ & & Ref \\
\hline Spanish & 661 & $479(72.47 \%)$ & $182(27.53 \%)$ & & $2.85(2.15-3.82)$ \\
\hline Insurance type, No. (\%) & & & & $<0.001$ & \\
\hline Private and other ${ }^{\mathrm{b}}$ & 212 & $179(84.43 \%)$ & $33(15.57 \%)$ & & Ref \\
\hline Public & 716 & $606(84.64 \%)$ & $110(15.36 \%)$ & & $0.98(0.65-1.52)$ \\
\hline Uninsured & 423 & $303(71.63 \%)$ & $120(28.37 \%)$ & & $2.15(1.42-3.33)$ \\
\hline High SDOH burden, No. (\%) & 204 & $164(80.39 \%)$ & $40(19.61 \%)$ & 1.00 & $1.01(0.69-1.46)$ \\
\hline Any positive SDOH, No. (\%) & 532 & $419(78.76 \%)$ & $113(21.24 \%)$ & 0.21 & $1.20(0.91-1.58)$ \\
\hline \multicolumn{6}{|c|}{ Individual SDOH measures, No. (\%) } \\
\hline Housing inadequacy & 171 & $139(81.29 \%)$ & $32(18.71 \%)$ & 0.87 & $0.95(0.62-1.41)$ \\
\hline Food insecurity & 381 & $293(76.90 \%)$ & $88(23.10 \%)$ & 0.04 & $1.36(1.02-1.82)$ \\
\hline Unreliable transportation & 110 & $93(84.54 \%)$ & $17(15.45 \%)$ & 0.32 & $0.74(0.42-1.23)$ \\
\hline Unstable utilities & 133 & $107(80.45 \%)$ & $26(19,55 \%)$ & 1.00 & $1.01(0.63-1.56)$ \\
\hline High residential $S D I^{\mathrm{C}}$ & 1174 & $931(79.30 \%)$ & $243(20.70 \%)$ & 0.004 & $2.05(1.29-3.43)$ \\
\hline
\end{tabular}

SDOH social determinants of health, SDI Social Deprivation Index

${ }^{a}$ Other race and ethnicity category includes American Indian or Alaskan Native, Native Hawaiian or Other Pacific Islander, Asian, Multiple Race, Refused or Unknown

bOther insurance type include Worker's Compensation and those listed as "other" in the medical record

${ }^{c}$ High Residential SDI indicates patient residing in a 5-digit Zip Code among highest quartile of social deprivation within Connecticut

${ }^{\mathrm{d}}$ Statistical difference for age was evaluated using a two-sided independent t-test, assuming unequal variances. Statistical difference in distributions of all other variables was evaluated using chi-square tests

relative to patients with English as their preferred language. In this adjusted model, insurance coverage, food insecurity, and high neighborhood SDI were not associated with significantly higher odds of SARS-CoV-2 detection.

For the matching analysis, we matched 210 participants with an English language preference to 269 participants with a Spanish language preference (35.5\% of total sample). Because exact matching was used to balance covariates, we achieved a standardized mean difference of 0 for all covariates included in the matching process (age, sex, race and ethnicity, insurance type, and high residential SDI). Weights generated during the matching process were used in the subsequent logistic regression to test the bivariate association of Spanish preferred language to SARS-CoV-2 detection among the matched sample. In this sample, we found a higher risk of SARS-CoV-2 detection associated with
Spanish language preference (OR 2.02, 95\% CI 1.27-3.27) consistent with our previous regression analysis (Table 2).

Both the adjusted logistic regression analysis and matching analysis produced similar odds with overlapping confidence bounds associated with Spanish as a preferred language after controlling for a range of other sociodemographic factors (Fig. 2).

\section{Discussion}

In this retrospective cohort study of over 1,300 individuals seeking walk-up testing for SARS-COV-2 by PCR at a community health center, we identified disparities in SARS-CoV-2 by ethnicity and by preferred language after adjusting for $\mathrm{SDOH}$, demographics, and neighborhood-level 
Fig. 1 SARS-CoV-2 Detection Rates by Social Determinants of Health (SDOH) Exposures among Study Cohort $(\mathrm{N}=1351)$.

\section{SARS-CoV-2 Detection Rate by SDOH Exposure}

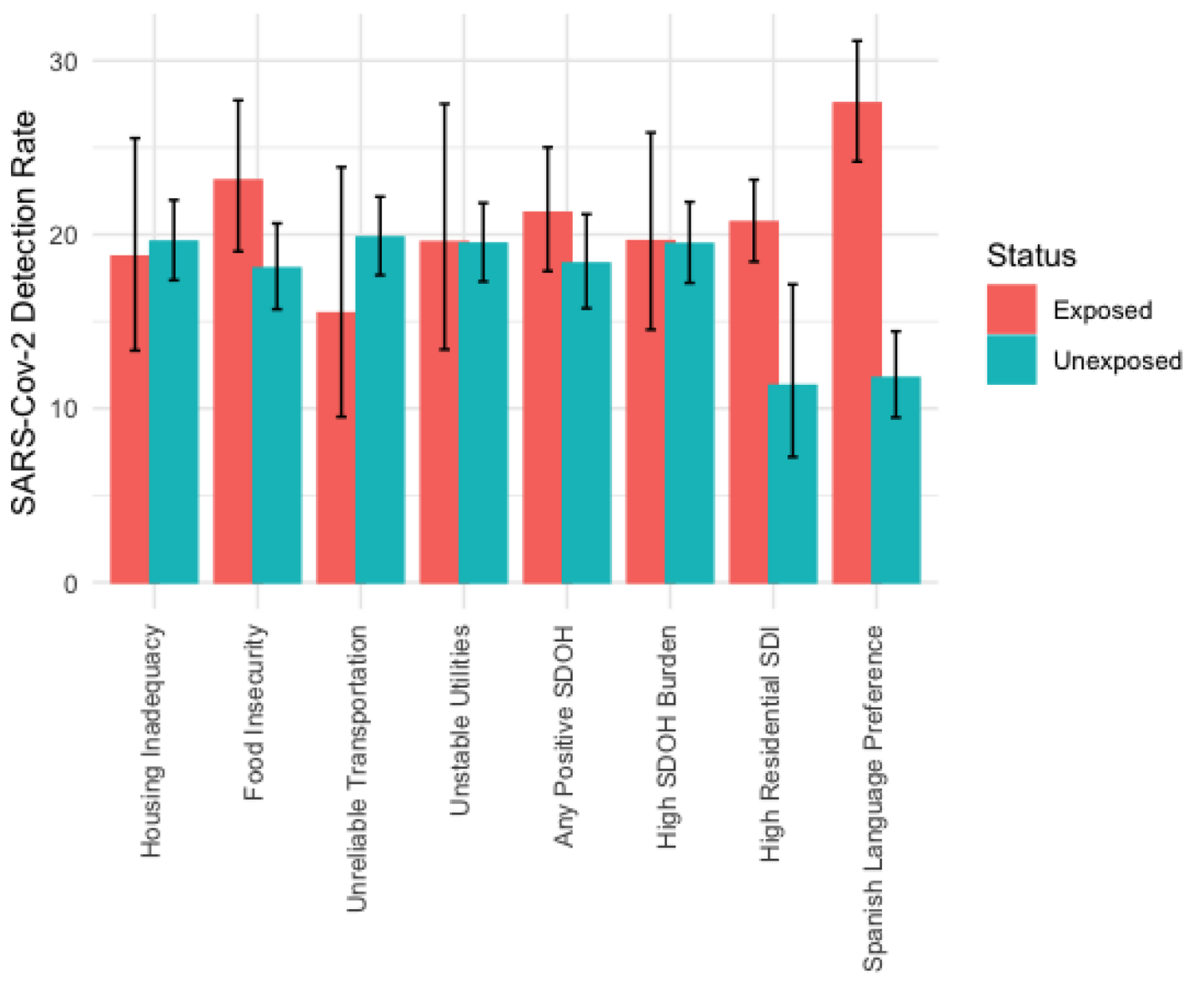

Note: Error bars display 95\% confidence intervals.

characteristics. The participants in our study demonstrated a high SDOH burden (nearly $40 \%$ reported at least one $\mathrm{SDOH}$ ) and had other features characteristic of medically underserved populations, including a majority who were not non-Hispanic White $(88 \%)$ and a high percentage who were either publicly insured (53\%) or uninsured (31\%). Preferring to receive care in Spanish was associated with nearly three times the odds of SARS-CoV-2 infection in our unadjusted analysis and roughly two times the odds of detection in both adjusted and matched analyses. Spanish language preference may plausibly be a proxy for recent immigration, age at immigration, undocumented immigration status, or educational attainment among immigrants [23]. The observed risk difference between language preference groups may relate to occupation in public-facing settings and/or settings that lack the capacity to comply with standards of infection control [24]. The association may be mediated by additional antiimmigrant structural stigma including multilevel discrimination, threats of detention or deportation, and limited access to health resources and economic relief [25].

Our findings extend the literature on the impact of social and structural determinants on SARS-CoV-2 infection [26, 27], hospitalization [28], and mortality [29, 30]. Racial and ethnic disparities in SARS-CoV-2 risk are well documented in COVID-19, suggesting that Black and Hispanic individuals are at greater risk for infection and poor outcomes [27, 29, 31]. Specific social and structural determinants have been identified as risk factors for infection and worse outcomes, such as homelessness [4] or school closures [32]. Regarding neighborhood- and community-level factors, a prior cross-sectional study in Milwaukee, Wisconsin, USA, adjusted for area deprivation and did not find this to impact SARS-CoV-2 infection or severity of disease [27]. Strengths of our analysis are that it employed a standardized SDOH screening instrument, self-reported race/ethnicity data, and a well-acknowledged measure of area social deprivation.

While the SDOH measured in our study were not associated with SARS-CoV-2 infection, the importance of SDOH in the burden of COVID-19 requires further study. This finding may be due to limited variation in SDOH burden within our study sample. Further, our analysis focused on selfreported individual-level $\mathrm{SDOH}$, which may obscure larger, structural determinants that impact the burden of COVID19 , including "lock-down" implementation, immigration policies, and systemic racism [33, 34]. Individual-level SDOH may predict worse outcomes among those already infected with COVID-19; but this is outside the scope of this analysis.

Our study has several limitations worth noting. First, participants represented a convenience sample of those seeking 
Table 2 Association of Select Social Determinants of Health and COVID-19 Detection

\begin{tabular}{ll}
\hline Characteristic & $\begin{array}{l}\text { Adjusted Odds (95\% } \\
\text { Confidence Interval) }\end{array}$ \\
\hline Age & $0.99(0.98-1.00)$ \\
Sex & \\
Male & $1.05(0.79-1.40)$ \\
Female & Ref \\
Race and ethnicity & \\
Hispanic & Ref \\
Non-Hispanic Black & $1.27(0.73-2.17)$ \\
Non-Hispanic White & $0.18(0.05-0.46)$ \\
Other & $0.73(0.27-1.71)$ \\
Language & \\
English & Ref \\
Spanish & $2.04(1.43-2.96)$ \\
Insurance type & \\
Private and other & \\
Public & Ref \\
Uninsured & $0.76(0.49-1.19)$ \\
FOOD INSECURITY & $1.23(0.78-1.98)$ \\
HIGH RESIDENTIAL SDI & $1.10(0.81-1.49)$ \\
\hline
\end{tabular}

SDOH social determinants of health

${ }^{a}$ Other insurance type include Worker's Compensation and those listed as "other" in the medical record

${ }^{b}$ Other race and ethnicity category includes American Indian or Alaskan Native, Native Hawaiian or Other Pacific Islander, Asian, Multiple Race, Refused or Unknown

${ }^{\mathrm{c}}$ High Residential SDI indicates patient residing in a 5-digit Zip Code among highest quartile of social deprivation within Connecticut

testing and may have oversampled among those who do not work during testing hours and/or are well enough and have resources to attend testing. Second, our sample may be biased towards those with greater SDOH burden as AHC tool completion was optional for those seeking SARS-CoV-2 testing. However, the demographic makeup of the sample is similar to that of the health center's patient population [35]. Third, race and ethnicity were self-reported during clinical care. Fourth, the study is based on the experience of a single health system, which may limit its generalizability to other geographic areas. Finally, the AHC tool does not capture potentially relevant $\mathrm{SDOH}$ factors such as household size and composition, residential density, and overall financial insecurity.

\section{New Contributions to the Literature}

The results of this study indicate the potential role of preferred language, and reinforce the importance of race and ethnicity, in the distribution of COVID-19 among a community health center cohort. The Healthy People 2030 Social Determinants of Health framework is organized into 5 placebased domains, including economic stability, education, health and health care, neighborhood and built environment, and social/community context [20]. Our findings suggest that preferred language, which may be a proxy for recent or age of immigration, undocumented status, or education levels, may be a target of the social/community context domain in public health COVID-19 prevention, mitigation, and treatment strategies. Outreach and resource allocation to those of racial and ethnic minorities, and those who prefer to receive care in a language other than English, should be prioritized in public health interventions. 
Fig. 2 Associations of Sociodemographic Variables with COVID-19 Detection

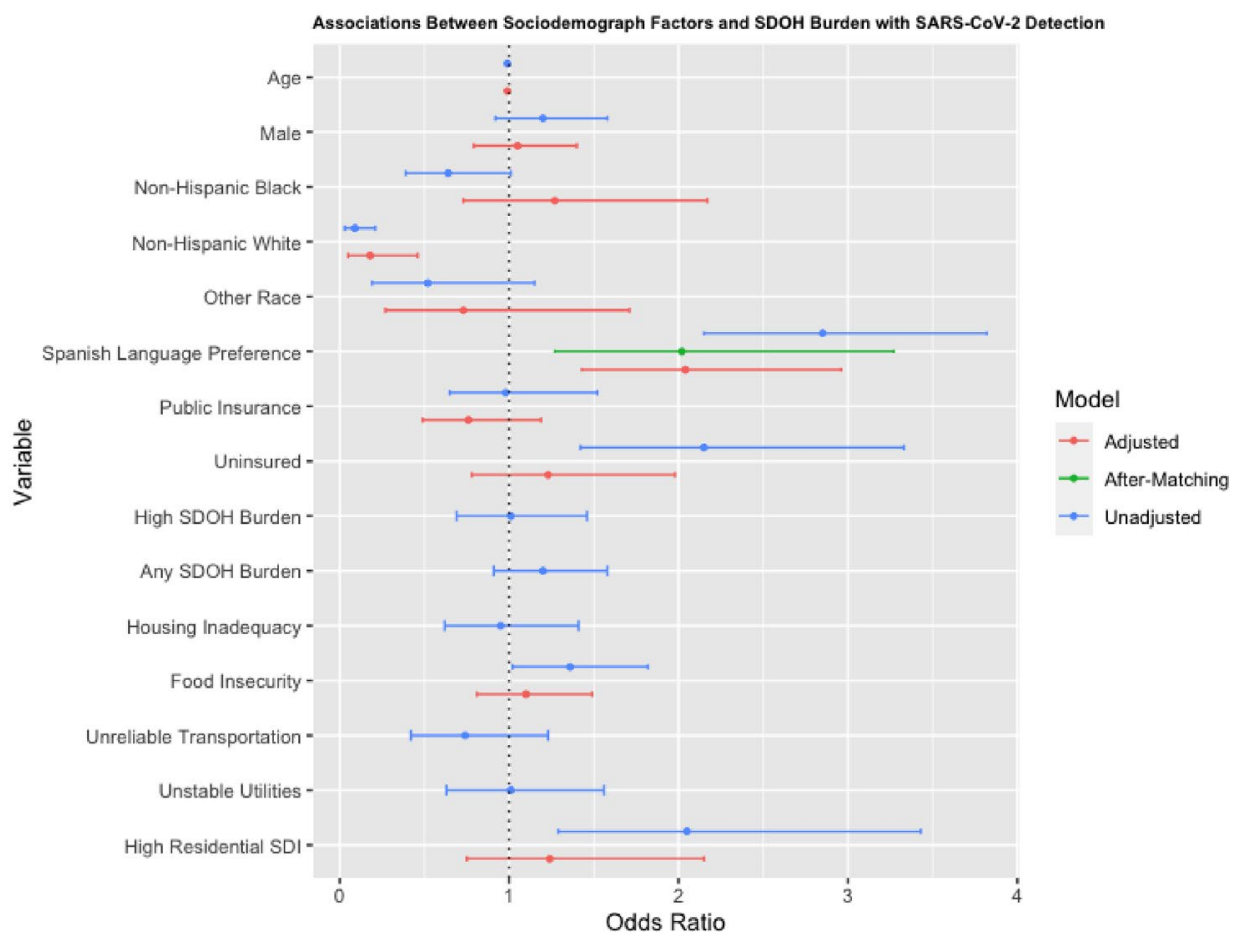

Note: Age is a continuous variable. Reference groups are omitted from the figure. The reference group for the sex variable is Female, the race and ethnicity variable is Hispanic, the language preference variable is English, and the insurance coverage variable is Private and Other. $\mathrm{N}=1361$ for adjusted and unadjusted logistic regression models. $\mathrm{N}=481$ for the after-matching logistic regression model.

\section{References}

1. Resnick BA, Mui PC, Bowie J, Kanchanaraksa S, Golub E, Sharfstein JM. The COVID-19 pandemic: an opportunity to transform higher education in public health. Public Health Rep. 2021;136:23-6.

2. Lopez L, Hart LH, Katz MH. Racial and Ethnic Health Disparities Related to COVID-19. JAMA. 2021;325:719.

3. Dunn CG, Kenney E, Fleischhacler SE, Bleich SN. Feeding lowincome children during the Covid-19 pandemic. N Engl J Med. 2020;382:40.

4. Tsai J, Wilson M. COVID-19: a potential public health problem for homeless populations. Lancet Public Health. 2020;5:e186-7.

5. Williams DR, Cooper LA. COVID-19 and health equity-a new kind of "herd immunity." JAMA. 2020;323:2478-80.

6. Bailey ZD, Krieger N, Agenor M, Graves J, Linos N, Bassett MT. Structural racism and health inequities in the USA: evidence and interventions. Lancet. 2017;389:1453-63.

7. Zhang M, Gurung A, Anglewicz P, Yun K. COVID-19 and immigrant essential workers: Bhutanese and Burmese refugees in the United States. Public Health Rep. 2021;136:117-23.

8. Van Lancker W, Parolin Z. COVID-19, school closures, and child poverty: a social crisis in the making. Lancet Public Health. 2020;5:e243-4.

9. Cm K, Oral E, Straif-Bourgeois S, Rung AL, Peters ES. The effect of area deprivation on COVID-19 risk in Louisiana. PLoS ONE. 2020;15:e243028.
10. Lewer D, Braithwaite I, Bullock M, Eyre MT, White PJ, Aldridge RW, et al. COVID-19 among people experiencing homelessness in England: a modelling study. Lancet Respir Med. 2020;8:1181-91.

11. Maness SB, Merrell L, Thompson EL, Griner SB, Kline N, Wheldon C. Social determinants of health and health disparities: COVID-19 exposures and mortality among African American people in the United States. Public Health Rep. 2021;136:18-22.

12. Metzl JM, Maybank A, De Maio F. Responding to the COVID19 pandemic: the need for a structurally competent health care system. JAMA. 2020;324:231-2.

13. National Association of Community Health Centers. About Health Centers; 2019.

14. Nocon RS, Lee SM, Sharma R, Ngo-Metzger Q, Mukamel DB, Gao Y, et al. Health care use and spending for medicaid enrollees in federally qualified health centers versus other primary care settings. Am J Public Health. 2016;106:1981-9.

15. Geiger HJ. The first community health center in Mississippi: communities empowering themselves. Am J Public Health. 2016;106:1738-40.

16. Council on Community Pediatrics. Poverty and Child Health in the United States. Pediatrics. 2016;2016:137.

17. American Academy of Family Physicians. Social Determinants of Health [Policy Statement]; 2013.

18. American College of Obstetricians and Gynecologists. Importance of social determinants of health and cultural awareness in the delivery of reproductive health care; 2018. 
19. Alderwick H, Gottlieb LM. Meanings and misunderstandings: a social determinants of health Lexicon for health care systems. Milbank Q. 2019;97:407-19.

20. U.S. Department of Health and Human Services. Social determinants of health framework. In; 2020.

21. Billioux A, K. Verlander, S. Anthony, Alley D. Standardized screening for health related social needs in clinical settings. National Academy of Medicine 2017.

22. Butler DC, Petterson S, Phillips RL, Bazemore AW. Measures of social deprivation that predict health care access and need within a rational area of primary care service delivery. Health Serv Res. 2013;48:539-59.

23. Young MT, Madrigal DS. Documenting legal status: a systematic review of measurement of undocumented status in health research. Public Health Rev. 2017;38:26.

24. Ahonen EQ, Fujishiro K, Cunningham T, Flynn M. Work as an inclusive part of population health inequities research and prevention. Am J Public Health. 2018;108:306-11.

25. Morey BN. Mechanisms by which anti-immigrant stigma exacerbates racial/ethnic health disparities. Am J Public Health. 2018;108:460-3.

26. Bambra C, Riordan R, Ford J, Matthews F. The COVID-19 pandemic and health inequalities. J Epidemiol Community Health. 2020;74:964-8.

27. Munoz-Price LS, Nattinger AB, Rivera F, Hanson R, Gmehlin CG, Perez A, et al. Racial disparities in incidence and outcomes among patients with COVID-19. JAMA Netw Open. 2020;3:e2021892.

28. Smati H, Cohen PA, Nagda DV, Saravanan Y, Kalugin PN, Li $\mathrm{CY}$, et al. Risk factors for hospitalization among patients with
COVID-19 at a community ambulatory clinic in Massachusetts during the initial pandemic surge. J Immigr Minor Health. 2021;23:1110-5.

29. Gross CP, Essien UR, Pasha S, Gross JR, Wang SY, NunezSmith M. Racial and ethnic disparities in population-level Covid-19 mortality. J Gen Intern Med. 2020;35:3097-9.

30. Paremoer L, Nandi S, Serag H, Baum F. Covid-19 pandemic and the social determinants of health. BMJ. 2021;372:129.

31. Martinez DA, Hinson JS, Klein EY, Irvin NA, Saheed M, Page $\mathrm{KR}$, et al. SARS-CoV-2 positivity rate for Latinos in the Baltimore-Washington, DC region. JAMA. 2020;324:392.

32. Van Lancker W, Parolin Z. COVID-19, school closures, and child poverty: a social crisis in the making. Lancet Public Health. 2020;5:243.

33. Prasad V, Sri BS, Gaitonde R. Bridging a false dichotomy in the COVID-19 response: a public health approach to the "lockdown" debate. BMJ Glob Health. 2020;2020:5.

34. Buse K, Nilo A, Kim J, Heywood M, Acaba J. COVID-19 combination prevention requires attention to structural drivers. Lancet. 2020;396:466.

35. Health Resources \& Services Administration Health Center Program. Fair Haven Community Health Clinic, Inc; Health Center Program Awardee Data. In. Washington, DC: Health Resources \& Services Administration; 2017.

Publisher's Note Springer Nature remains neutral with regard to jurisdictional claims in published maps and institutional affiliations. 